\title{
A NEW UPPER BOUND FOR THE IRREGULARITY STRENGTH OF GRAPHS
}

\author{
MACIEJ KALKOWSKI, MICHAE KAROŃSKI, AND FLORIAN PFENDER
}

\begin{abstract}
A weighting of the edges of a graph is called irregular if the weighted degrees of the vertices are all different. In this note we show that such a weighting is possible from the weight set $\left\{1,2, \ldots, 6\left\lceil\frac{n}{\delta}\right\rceil\right\}$ for all graphs not containing a component with exactly 2 vertices or two isolated vertices.
\end{abstract}

\section{INTRODUCTION}

All graphs in this note are finite and simple. For notation not defined here we refer the reader to [4].

For some $k \in \mathbb{N}$, let $\omega: E(G) \rightarrow\{1,2, \ldots, k\}$ be an integer weighting of the edges of a graph $G$. This weighting is called irregular if the weighted degrees $d_{\omega}(v)=\sum_{u \in N(v)} \omega(u v)$ of the vertices are all different. It is easy to see that for every graph $G$ which has at most one isolated vertex and no component isomorphic to $K^{2}$, there exists an irregular weighting for some smallest $k$, the irregularity strength $s(G)$ of $G$. If $G$ contains a $K^{2}$ or multiple isolated vertices, we set $s(G)=\infty$.

The irregularity strength was introduced in [2] by Chartrand et al. . For all graphs with $n:=|G|>3$ and $s(G)<\infty$, Nierhoff [8] showed the tight bound $s(G) \leq n-1$, extending a result by Aigner and Triesch [1]. Faudree and Lehel considered regular graphs in [5]. They showed that if $G$ is $d$-regular $(d \geq 2)$, then $\left\lceil\frac{n+d-1}{d}\right\rceil \leq s(G) \leq\left\lceil\frac{n}{2}\right\rceil+9$, and they conjectured that $s(G) \leq\left\lceil\frac{n}{d}\right\rceil+c$ for some constant $c$.

A first bound involving the minimum degree $\delta$ was given by Frieze et al. in [6] where they showed that $s(G) \leq 60\left\lceil\frac{n}{\delta}\right\rceil$, for graphs with maximum degree $\Delta \leq n^{1 / 2}$. For graphs with high minimum degree, Cuckler and Lazebnik showed that $s(G) \leq 48\left\lceil\frac{n}{\delta}\right\rceil+6$ in [3]. Finally, Przybyło showed in [10] that $s(G) \leq 112 \frac{n}{\delta}+28$ for general graphs and in [9] that $s(G) \leq 16 \frac{n}{d}+6$ for $d$-regular graphs.

In this note we give a construction improving the bounds stated in the previous paragraph. We use ideas similar to the ones used in [7].

1991 Mathematics Subject Classification. 05C78, (05C15).

Key words and phrases. irregular graph labelings. 
Theorem 1. Let $\delta$ be the minimum degree of $G$ and $n=|G|$. If $s(G)<\infty$, then $s(G) \leq 6\left\lceil\frac{n}{\delta}\right\rceil$.

Considering the sharpness of these results, no graphs classes with $s(G)>\left\lceil\frac{n}{\delta}\right\rceil+c$ are known to us, similarly to the case of regular graphs mentioned above.

\section{ProOF}

Since $s(G) \leq n-1$, there is nothing to prove for $\delta \leq 6$, so we may assume that $\delta \geq 7$. Order the vertices $v_{1}, v_{2}, \ldots, v_{n}$ such that for $1 \leq i<k \leq j \leq n$, whenever $v_{i}$ and $v_{j}$ belong to the same component of $G$,

- $v_{k}$ also belongs to that component of $G$, and

- $v_{i}$ has a neighbor $v_{\ell}$ with $\ell>i$.

Going through the vertices in order, we will assign two weights $\omega_{1}$ and $\omega_{2}$ to each edge $v_{i} v_{j}$ (where $i<j$ ), and $\omega\left(v_{i} v_{j}\right)=\omega_{1}\left(v_{i} v_{j}\right)+\omega_{2}\left(v_{i} v_{j}\right)$. The first weight $\omega_{1}\left(v_{i} v_{j}\right) \in\left\{1,2, \ldots, 2\left\lceil\frac{n}{\delta}\right\rceil\right\}$ is assigned when we process $v_{i}$, the second weight $\omega_{2}\left(v_{i} v_{j}\right) \in\left\{0,2\left\lceil\frac{n}{\delta}\right\rceil, 4\left\lceil\frac{n}{\delta}\right\rceil\right\}$ is initially set to $2\left\lceil\frac{n}{\delta}\right\rceil$ and finalized when we process $v_{j}$.

Let

$$
\mathcal{W}:=\left\{\left\{a+4 b\left\lceil\frac{n}{\delta}\right\rceil, a+(4 b+2)\left\lceil\frac{n}{\delta}\right\rceil\right\} \mid a, b \in \mathbb{Z}, 0 \leq a \leq 2\left\lceil\frac{n}{\delta}\right\rceil-1\right\}
$$

be a set of disjoint pairs of integers covering $\mathbb{Z}$, and for a given $\omega$ and $1 \leq i \leq n$, let $W\left(v_{i}\right) \in \mathcal{W}$ be the unique pair containing $d_{\omega}\left(v_{i}\right)$.

Let $X$ be the set of indices $i$, such that either $v_{i}$ or $v_{i+1}$ is the final vertex of a component. For $i \leq n$, assume that all vertices $v_{k}$ with $k<i$ have been considered already.

If $i \notin X$, we want to adjust $\omega$ such that $W\left(v_{i}\right) \neq W\left(v_{k}\right)$ for all $k<i$. In the remainder of the construction, $W\left(v_{i}\right)$ will not change anymore. Reserving a pair of values for $d_{\omega}\left(v_{i}\right)$ like this gives us the freedom to later adjust $\omega_{2}\left(v_{i} v_{j}\right)$ for $j>i$ without creating a conflict.

To this end, we can freely choose $\omega_{1}\left(v_{i} v_{j}\right)$ for $i<j$ and choose $\omega_{2}\left(v_{k} v_{i}\right)$ for $k<i$ from one of the two values keeping $d_{\omega}\left(v_{k}\right)$ in $W\left(v_{k}\right)$. If $v_{i}$ has $d^{+} \geq 1$ neighbors $v_{j}$ with $j>i$ and $d^{-}$neighbors $v_{k}$ with $k<i$, this gives us

$$
2\left\lceil\frac{n}{\delta}\right\rceil\left(d^{+}+d^{-}\right)-d^{+} \geq 2 n-d^{+}>2 i
$$

consecutive options for $d_{\omega}\left(v_{i}\right)$. These options intersect more than $i$ pairs of $\mathcal{W}$. At most $i-1$ of these pairs can already be used as some $W\left(v_{k}\right)$ by a neighbor $v_{k}$ of $v_{i}$ with $k<i$, so we can find the desired pair $W\left(v_{i}\right)$, together with a preliminary weighting $\omega$. 
If $\{i, i+1\} \subseteq X$, note that $v_{i} v_{i+1}$ is an edge. We may choose $\omega_{1}\left(v_{i} v_{i+1}\right)$ such that no three vertices $v_{j}$ with $j \in X$ and $j \leq i+1$ have the same weight $\omega_{1}$ as there are less than $\left\lceil\frac{n}{\delta}\right\rceil$ components and thus $|X|<2\left\lceil\frac{n}{\delta}\right\rceil$.

Let $j \in\{i, i+1\} \subseteq X$. We want to adjust $\omega_{2}\left(v_{k} v_{j}\right)$ for edges with $k<i$ so that all weighted degrees $d_{\omega}\left(v_{\ell}\right)$ for $\ell \leq j$ are different. At this stage we allow that $W\left(v_{j}\right)=W\left(v_{\ell}\right)$ for one $\ell<j$, since both $d_{\omega}\left(v_{j}\right)$ and $d_{\omega}\left(v_{\ell}\right)$ are finalized in this step as they don't have neighbors $v_{s}$ with $s>i+1$. There are at least $\delta-1$ neighbors $v_{k}$ of $v_{j}$ with $k<i$. As we have picked all the $W\left(v_{k}\right)$ after finalizing $d_{\omega}\left(v_{s}\right)$ for all $s \in X$ with $s<i$, at most one of the pairs $W\left(v_{k}\right)$ may contain the weighted degrees of two vertices (namely, $d_{\omega}\left(v_{k}\right)$ and $d_{\omega}\left(v_{i}\right)$ if $j=i+1$ ). Thus, we may adjust $\omega_{2}\left(v_{k} v_{j}\right)$ on all these edges but possibly one, keeping $d_{\omega}\left(v_{k}\right) \in W\left(v_{k}\right)$.

This gives us $\delta-1$ options for $d_{\omega}\left(v_{j}\right)$, an arithmetic progression with step size $2\left\lceil\frac{n}{\delta}\right\rceil$. These options completely contain at least $\frac{\delta-3}{2} \geq 2$ pairs in $\mathcal{W}$. At most one such pair may contain some $d_{\omega}\left(v_{\ell}\right)$ with $j>\ell \in X$ by our choice of $\omega_{1}\left(v_{i} v_{i+1}\right)$, so there is a pair left which does not contain such a weighted degree. At most one vertex $v_{\ell}$ with $j>\ell \notin X$ can have its weighted degree in that pair. Adjust the weights $\omega_{2}\left(v_{k} v_{j}\right)$ so that $d_{\omega}\left(v_{j}\right)$ is in that pair. If now $d_{\omega}\left(v_{j}\right)=d_{\omega}\left(v_{\ell}\right)$, we may either change some weight $\omega_{2}\left(v_{k} v_{j}\right)$ with $k \neq \ell$ to move $d_{\omega}\left(v_{j}\right)$ to the other value in that pair, or change both $\omega_{2}\left(v_{k} v_{j}\right)$ and $\omega_{2}\left(v_{\ell} v_{j}\right)$ to keep $d_{\omega}\left(v_{j}\right)$ and to move $d_{\omega}\left(v_{\ell}\right)$ to the other value in that pair (which may be necessary if $\left.v_{\ell} v_{j} \in E\right)$. This concludes the proof.

\section{REFERENCES}

1. M. Aigner and E. Triesch, Irregular assignments of trees and forests. SIAM J. Discrete Math. 3(1990), 439-449.

2. G. Chartrand, M.S. Jacobson, J. Lehel, O.R. Oellermann, S. Ruiz, F. Saba, Irregular Networks. Congressus Numerantium 64 (1988), 187 - 192.

3. B. Cuckler and F. Lazebnik, Irregularity Strength of Dense Graphs, J. Graph Theory 58 (2008), 299-313.

4. R. Diestel, "Graph Theory," Springer Verlag, Heidelberg, 2005.

5. R.J. Faudree and J. Lehel, Bound on the irregularity strength of regular graphs. Colloq. Math. Soc. János Bolyai, 52, Combinatorics, Eger.

North Holland, Amsterdam, 1987, 247-256.

6. A. Frieze, R. Gould., M. Karoński, F. Pfender, On Graph Irregulaity Strength, J. Graph Theory 41 (2002), 120-137.

7. M. Kalkowski, M. Karoński, F. Pfender, Vertex-Coloring Edge Weightings: Towards the 1-2-3-Conjecture, J. Combin. Theory (B), to appear.

8. T. Nierhoff, A tight bound on the irregularity strength of graphs. SIAM J. Discrete Math. 13 (2000), 313-323. 
9. Przybyło, J. Irregularity strength of regular graphs, Electronic J. Combinatorics 15 (2008) (1)\#R82

10. Przybyło J., Linear bound for on the irregularity strength and the total vertex irregularity strength of graphs, SIAM J. Discrete Math., to appear.

Current address, M. Kalkowski: Adam Mickiewicz University, Faculty of Mathematics and Computer Science, Poznań, Poland

E-mail address: kalkos@atos.wmid.amu.edu.pl

Current address, M. Karoński: Adam Mickiewicz University, Faculty of Mathematics and Computer Science, Poznań, Poland, and Emory University, Department of Mathematics and Computer Science, Atlanta, GA, USA

E-mail address: karonski@amu.edu.pl

Current address, F. Pfender: Universität Rostock, Institut für Mathematik, Rostock, Germany

E-mail address: Florian.Pfender@uni-rostock.de 